\title{
Development of a portable Escherichia coli quantifier device
}

\begin{abstract}
Healthcare-associated infection is one of the main causes of mortality and healthcare costs increase. Microorganism's identification and counting are essential for human health preservation. Escherichia coli is an important bacterium, which causes Healthcareassociated infection. Spectrophotometry method can be used to Escherichia coli counting, but the ordinary devices are not portable and are very expensive. The aim of this study is to develop a portable and low-cost device to quantifier the number of Escherichia coli 's cells per $\mathrm{mL}$ based on Lambert-Beer law. Therefore, three steps were performed: device construction, software development and device testing. In the prototype test, a comparison was made between three measurement processes: concentration analysis results by visually counting cells; counting with a conventional spectrophotometer; and counting with a developed quantifier device. As conclusion, E. Coli quantifier device has some advantages: low cost, small size, it automatically performs 400 measurements of each sample to calculate the average and it is able to communicate instantly with a computer to facilitate interpretation of the results.
\end{abstract}

Keywords: count of microorganisms, Escherichia coli, analytical instrumentation
Volume 5 Issue I - 2019

\author{
Filipe Loyola Lopes, Bruna Tavares Leite, \\ Anderson Fagiani Fraga, Elisa Rennó \\ Carneiro Dester, Francisco Eduardo de \\ Carvalho Costa
}

National Institute of Telecommunication - Inatel, Brazil

Correspondence: Filipe Loyola Lopes, National Institute of Telecommunication - Inatel. Av. João de Camargo, 5 I 0, Centro, 37540-000, Santa Rita do Sapucai, Minas Gerais, Brazil, Email filipeloyola@geb.inatel.br

Received: December 29, 2018 | Published: January 23, 2019
Abbreviations: ATCC, American Type Culture Collection; COM, Serial communication computer portal; E. Coli, Escherichia coli; EMB Agar, Methyl Eosin Agar; Inatel, National Institute of Telecommunication; USB, Universal Serial Bus

\section{Introduction}

Healthcare-associated infection is a serious public health problem, because it is responsible for raising the mortality rate and healthcare costs worldwide. ${ }^{1}$ Identifying contaminations caused by microorganisms, like Escherichia coli (E. Coli), is essential to preserve human health, but hospitals can be as reservoirs for viruses, fungi, bacteria and spores. ${ }^{2}$ Bacteria, in special, is part of the human flora and may pose health risks, especially for individuals with compromised clinical status, for example $E$. Coli can cause Nosocomial urinary tract infection..$^{3-6} E$. Coli is an anaerobic bacterium of bacillary morphology, Gram-Negative type. E. Coli is uropathogen in the fecal microbiota, which can colonize the urethral canal and ascend to the bladder, most frequent in women. ${ }^{3,7}$ The search for reduced time to microbiological analysis and technologies development results in new techniques to improve the analysis process and to minimize errors. ${ }^{8} \mathrm{Clinical}$ analyzes using physicochemical techniques, such as spectrophotometry, have benefited immensely from quantitative microbiological analysis methods. ${ }^{9}$ Spectrophotometry has theoretical foundation in LambertBeer law and can be aid in medical decisions. ${ }^{10}$ Spectrophotometry is one of the most used qualitative and quantitative techniques due to the robustness and diversity of applications, however spectrophotometry typical devices is not portable and it is also expensive for applications in health care establishments with few financial resources. ${ }^{8-10}$ The cost of a simple spectrophotometer (model 225 D CELM-E) in Brazil was budgeted approximately 2,350.00 USD and its physical dimension is $385 \mathrm{~mm}$ (width), $285 \mathrm{~mm}$ (depth) and $215 \mathrm{~mm}$ (height). The aim of this study is to construct and test the E. Coli quantifier device. This device has microcontroller (chip with embedded programming), it is portable, low-cost and it can quantify cells of E. Coli bacteria in samples with different concentrations of this microorganism.

\section{Experimental methods}

The methodology of this research was divided into three stages: device construction, software development for computer communication and equipment testing.

\section{Device construction}

The development and construction of the device took place in the Medical Technologies Laboratory of the National Institute of Telecommunications (Inatel). The electronic boards were manually assembled and interconnected to the LauchPad MSP430F5529 (Texas Instruments) microcontroller platform (Figure 1). Power Source: Powered by USB standard (5 volts). LauchPad: Microcontroller platform (16-bit MSP430 25MHz), minimum hardware assembly for platform utilization and firmware development. Firmware development was done through layered programming using $\mathrm{C}$ language. Light Source: Choice of light source, infrared LED (IR LED) for monochrome beam production at $880 \mathrm{~nm}$ and development of drive driver circuit. Sample: The holder mechanical development to receive the sample cuvette of solute to be measured. Transduction circuit: Circuit with photodiode, signal conditioning (amplification and filtering). Man-machine interface: liquid crystal display with two lines and 16 columns, keyboard development, hardware and firmware development for keyboard and display control. Communication with computer: Communication between the device and a computer through a USB cable.

\section{Software development for computer communication}

It was developed in the GUIDE platform of Matlab Program (MathWorks Inc), available in Inatel, it allows to perform mathematical operations and to use graphical tools for the manipulation of digital signals. The communication between the computer and the equipment was performed through serial protocol with USB cable. To this end, it was necessary to divide the information into eight-bit packets. These packages are reassembled by the software when they 
arrive at the computer through the virtual COM port. The software objective is to establish instant communication with the $E$. Coli quantifier through USB cable in order to capture the data read from biological samples in order to facilitate the interpretation of the user through graphs and values. The software also performs simple linear regression of the sampled points, aiming to calculate the coefficient of determination $\left(\mathrm{R}^{2}\right)$ of the curve plotted from different concentrations of microorganisms.

\section{Equipment testing}

The test was performed by comparing the results between the developed device measurements, the spectrophotometer measurements CELM-E model 225 D provided by Inatel, and visual counting of bacteria colonies. In order to achieve the E. Coli concentration measurements, the samples were artificially contaminated (saline solution) from an American Type Culture Collection (ATCC) strain of E. Coli 25922, provided by SANOBIOL (Brazilian Industry). The culture was performed in a selective medium for enterobacteria, Methyl Eosin Agar (EMB Agar), and the logarithmic growth phase was used. The suspension of the microorganism was obtained at the concentration of $9 \mathrm{~mL}$ of saline solution, with turbidity corresponding to $1 \times 106(\mathrm{cell} / \mathrm{mL})$, according to the Macfarland scale. ${ }^{9}$ For each treatment, tubes with $5 \mathrm{~mL}$ of bacteria suspension were tested. Thereafter, each treatment was spread on the surface of different EMB agar plates through Drigalsk. The colonies grew for 48 hours at $35^{\circ} \mathrm{C}$ for three counting methods (Figure 2). Visual counting of the cell concentration (cells $/ \mathrm{mL}$ ) in the samples was performed through the image obtained by digital photography of the cultivated Petri dishes. The pen tool of the Paint program (Microsoft) was used for the counting. The analysis of the results was obtained using Matlab and Excel (Microsoft), using simple linear regression and coefficient of determination $\left(\mathrm{R}^{2}\right)$. The resulting data was analyzed separately, but always between two distinct groups.

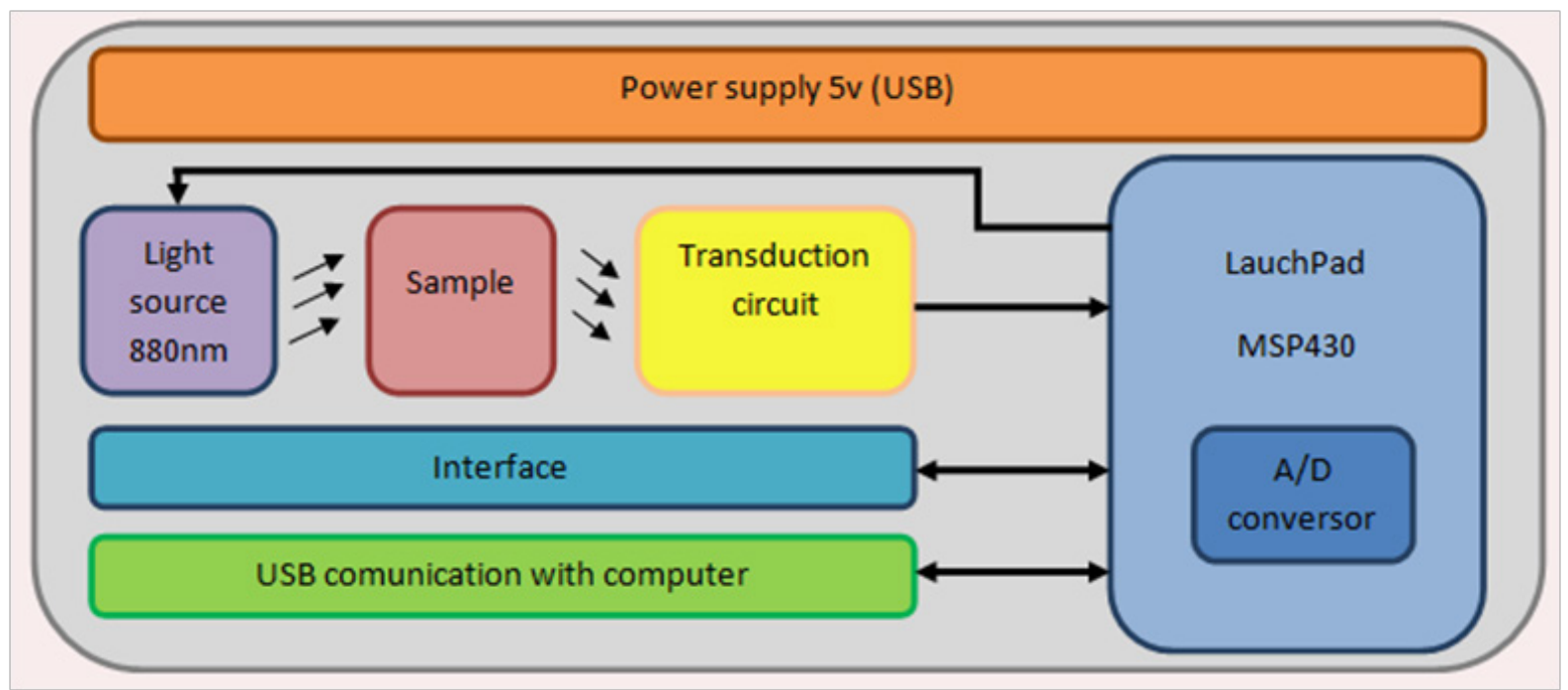

Figure I Block diagram of the E. Coli quantifier prototype.

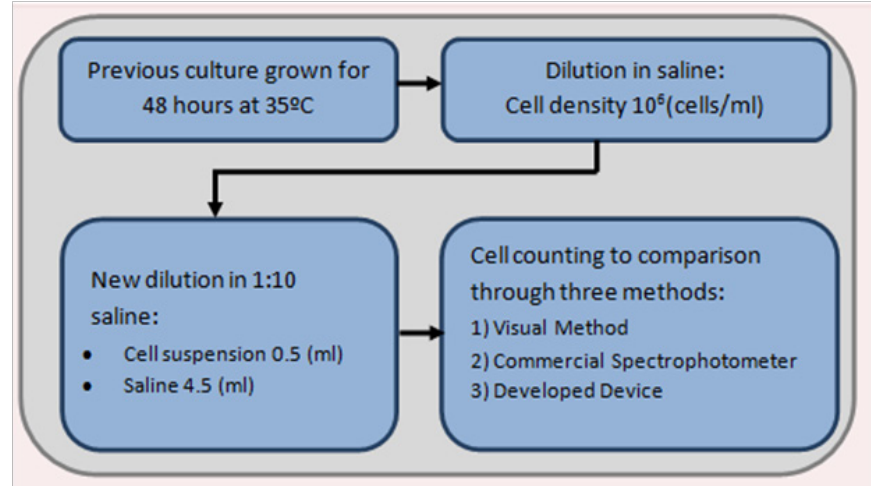

Figure 2 Flowchart of the $E$. Coli quantifier test process.

\section{Results}

The prototype was developed using low-cost and easily accessible components. Material price of prototype is 64.48 USD and this physical dimension is $107 \mathrm{~mm}$ (width), $205 \mathrm{~mm}$ (depth) and $40 \mathrm{~mm}$ (height) (Table 1) (Figure 3). The device can be calibrated. User can calibrate opaque sample $(0 \%$ transmittance $)$ and transparent sample (100\% transmittance distilled water or saline solution). The results can be graphically viewed on a computer screen (Figure 4). Developed program can check the stability of the readings performed, since the equipment was programmed to perform 400 repetitions of sample reading and then plot on a graph that must be constant, minimizing error margins. The software is able to mark " $\mathrm{n}$ " read points as the command is given by the user. With marked "n" points, the software offers the option of performing simple linear regression and calculation of the coefficient of determination $\left(\mathrm{R}^{2}\right)$ with just the click of a button. Visual counting of the cell concentration (cells $/ \mathrm{mL}$ ) in the samples was performed through the image obtained by digital photography of the cultivated Petri dishes. The results of visual counting can be found in Table 2 and an image of sample can be seen in Figure 5. Data obtained commercial spectrophotometer and device developed can be seen in the Table 3. The comparison between the data obtained from conventional spectrophotometer and visual counting result, as shown in Figure 6A \& Figure 6B, demonstrates the comparison between $E$. Coli counter and visual counting. From the graphs it was possible to apply the simple linear regression method and the calculation of the coefficient of determination $\left(\mathrm{R}^{2}\right)$. 

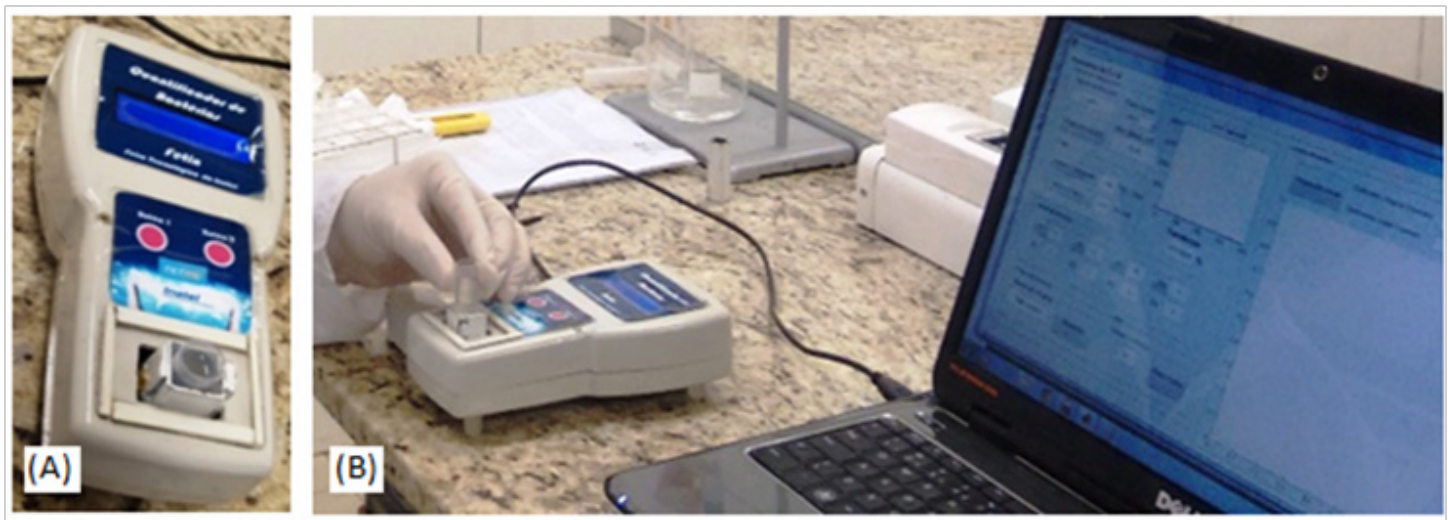

Figure 3 (A) Prototype of E. Coli quantifier and (B) prototype being used.

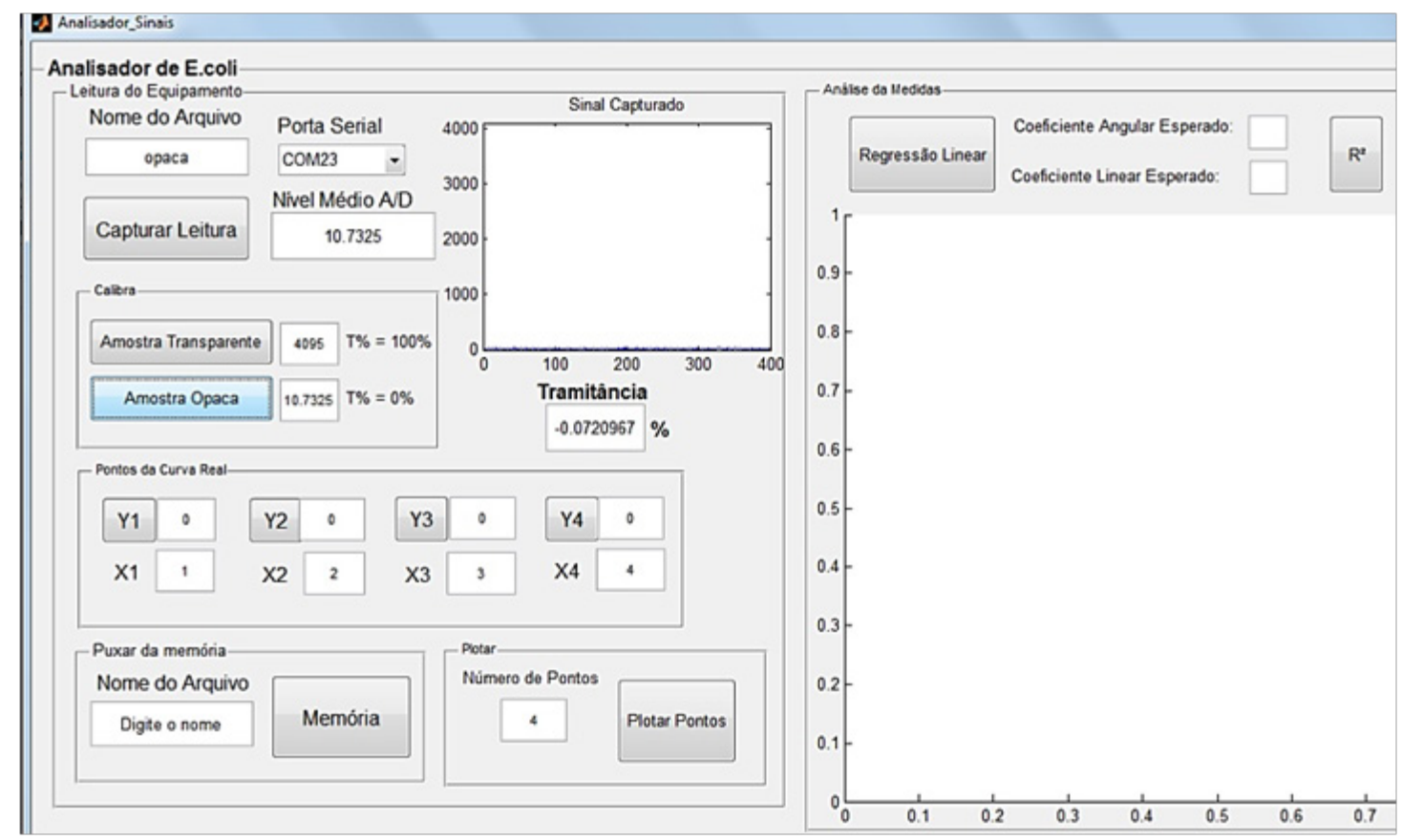

Figure 4 Application screen developed in the Matlab Program Guide tool for signal analysis.

Table I Cost of components in Brazil to assembly of the prototype.The values are in US Dollars (USD) with a price of 01.00 USD equal to 3.68 Brazilian Reais. In this analysis the labor costs was not considered

\begin{tabular}{llll}
\hline Description & Quantity & Unit price (USD) & Total price (USD) \\
\hline _MSP430F5529 LaunchPad Development Kit & 01 & 20.78 & 20.78 \\
Photodiode 880nm + import taxes & 01 & 2.56 & 2.56 \\
Infrared LED + import taxes & 01 & 3.02 & 3.02 \\
Display 16x2 & 01 & 8.62 & 8.62 \\
Op. Amp. LM741 & 01 & 0.52 & 0.52 \\
Plastic Box & 01 & 13.46 & 13.46 \\
Sample support & 01 & 13.59 & 13.59 \\
Printed circuit board & 01 & 1.93 & 1.93 \\
Total & & & 64.48 \\
\hline
\end{tabular}


Table 2 Result of the visual count

\begin{tabular}{ll}
\hline Escala Macfarland & Contagem visual \\
\hline $10^{0}$ & $60($ células $/ \mathrm{mL})$ \\
$10^{-1}$ & $30($ células $/ \mathrm{mL})$ \\
$10^{-2}$ & $20($ células $/ \mathrm{mL})$ \\
$10^{-3}$ & $30($ células $/ \mathrm{mL})$ \\
$10^{-4}-10^{-6}$ & nulo
\end{tabular}

Table 3 Comparison of measure with commercial spectrophotometer and $E$. Coli counter device, using Macfarland scale for dilution

\begin{tabular}{lll}
\hline Macfarland scale (Dilution) & $\begin{array}{l}\text { commercial } \\
\text { spectrophotometer }\end{array}$ & $\begin{array}{l}\text { device } \\
\text { developed }\end{array}$ \\
\hline Opaque sample & $0 \%$ & $0 \%$ \\
$10^{0}$ & $89.9 \%$ & $96.0519 \%$ \\
$10^{-1}$ & $99.9 \%$ & $99.0967 \%$ \\
$10^{-2}$ & $99.9 \%$ & $102.0102 \%$ \\
$10^{-3}$ & $100.7 \%$ & $99.9124 \%$ \\
$10^{-4}$ & $104.7 \%$ & $106.098 \%$ \\
$10^{-5}$ & $99.2 \%$ & $103.033 \%$ \\
$10^{-6}$ & $98 \%$ & $101.94 \%$ \\
Transparent sample & $100 \%$ & $100 \%$ \\
\hline
\end{tabular}

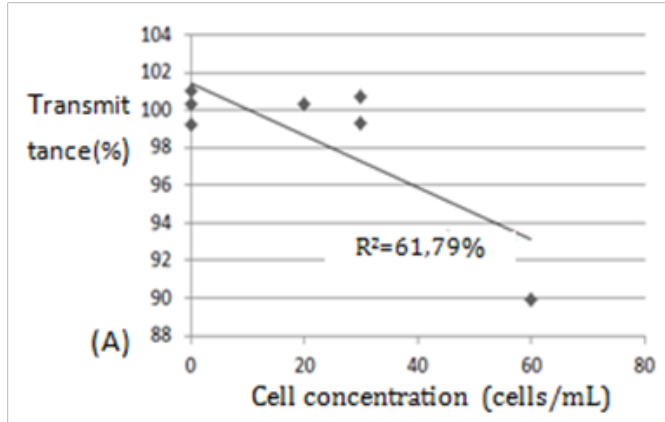

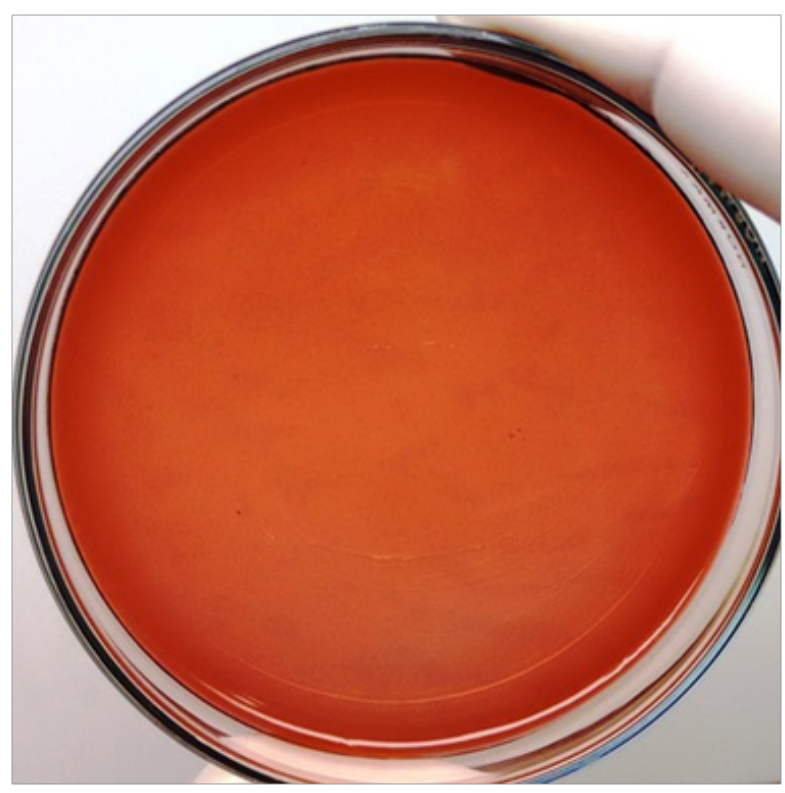

Figure 5 Photo of the petri dish where the E. Coli was grown.

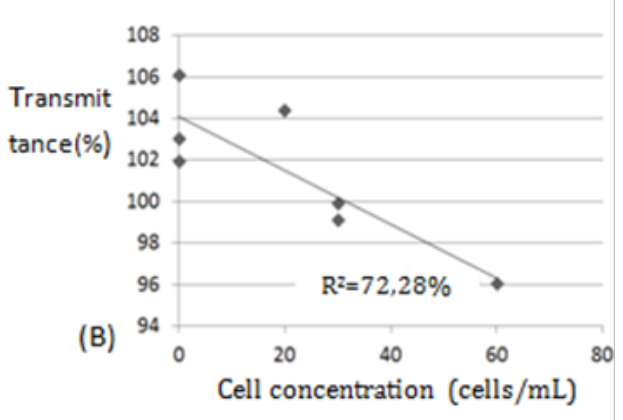

Figure 6 (A) Comparison between visual method count and conventional spectrophotometer. (B) Comparison between visual method and E. Coli counter.

\section{Discussion}

Identifying and controlling bacterial colonies in a hospital is very important to decrease mortality rate and healthcare costs. ${ }^{1}$ Analysis and the study of cases of microbiology using techniques such as spectrophotometry are extremely important. ${ }^{8,9}$ Low cost and portable equipment are very relevant for using in bio safety hospitals, food industries and other environments subject to $E$. Coli contamination. The spectrophotometer used as reference was budgeted approximately $2,350.00$ USD. The cost of the parts for the assembly of the prototype was 64.48USD. This comparison is not complete, since they do not constitute labor costs for prototype development or the cost of product certification. However, it may be a sign of price improvement. An important factor influencing the low cost of the prototype is that $E$. Coli quantifier uses specific wavelengths, minimizing the use of components for different wavelengths. In contrast, the commercial spectrophotometer has many applications and uses many wavelengths..$^{10}$ It is important to note that the equipment developed (107mm width, $205 \mathrm{~mm}$ depth and $40 \mathrm{~mm}$ height) was much smaller than commercial equipment $(385 \mathrm{~mm}$ width, $285 \mathrm{~mm}$ depth and $215 \mathrm{~mm}$ height).In the comparison between the data obtained from conventional spectrophotometer and E. Coli counter, the E. Coli quantifier device shows greater correlation $(72.28 \%)$ with visual measurement than conventional spectrophotometer (61.79\%), since the coefficient of determination $\left(\mathrm{R}^{2}\right)$ was higher, although in some points it presented less accuracy. It is important to note that the measurements were performed using a low concentration of microorganisms, which reveals a high sensitivity of both equipment tested and that the errors of accuracy presented by the prototype can be reduced for higher concentrations. Both methods showed a strong correlation between dependent and independent variables (higher than $60 \%)$. According to Pontes ${ }^{10}$ perfect correlation $\left(\mathrm{R}^{2}=100\right)$; very strong correlation $\left(90<\mathrm{R}^{2}<99\right)$; strong correlation $\left(60<\mathrm{R}^{2}<90\right)$; mean correlation $\left(30<\mathrm{R}^{2}<60\right)$; poor correlation $\left(10<\mathrm{R}^{2}<30\right)$; and null correlation $\left(\mathrm{R}^{2}<10\right) .{ }^{9}$ For future, it would be interesting new tests with higher concentrations of cells, using other wavelengths, other types of microorganisms and improvement of the firmware for the reduction of accuracy errors.

\section{Conclusion}

An E. Coli counter prototype was developed and underwent initial tests to compare measurements performed by the developed device and a conventional spectrophotometer. Both results were compared with the visual method, resulting in coefficient of determination of $72 \%$ and $62 \%$, respectively. The developed device has the advantages: higher correlation to visual method, low cost, reduced size (portable), 400 readings of each sample for calculation of average in order to 
reduce errors and communicates automatically with computer to facilitate biostatistician analysis. The device has the potential to assist the analysis of microorganisms in hospitals, clinics and companies. ${ }^{11}$

\section{Acknowledgments}

We thank Inatel for the use of laboratories, components and materials and a team of researchers.

\section{Conflicts of interest}

Author declares that there is no conflict of interest.

\section{References}

1. WHO. Health care-associated infection fact sheet. 2016.

2. Weber DJ, Anderson D, Rutala WA. The role of the surface environment in healthcare-associated infections. Curr Opin Infect Dis. 2013;26(4):338-344.

3. Merle V, Germain JM, Bugel H, et al. Nosocomial urinary tract infections in urology patients: assessment of a prospective surveillance program including 10,000 patients. European Urology. 2002;41:483-489.
4. Freire I. Infections related to health care in pediatrics. Revista Brasileira de Ciências da Saúde. 2013;11(35).

5. Costa F. Environmental contamination in a hospital by Pseudomonas aeruginosa, Klebsiella spp, Mycobacterium non tuberculosis and other Opportunistic Microorganisms. 2013.

6. Paula Júnior, José Dionísio. Identification and sensitivity profile of bacteria on tourniquets used in hospitals. Journal of Basic and Applied Pharmaceutical Sciences. 2013;34(2):269-273.

7. Cunha AHN, Vieira JA. Detection of escherichia coli in wastewater using flow system for turbidimetry. Revista Mirante. 2014;7(2).

8. Souza FVG. Design of a portable spectrophotometer in the visible spectrum. 2014.

9. Rocha FRO, Teixeira LSG. Strategies to increase sensitivity in uv-vis spectrophotometry. Química Nova, 2004;27:807-812.

10. Pontes AS. Development of a LED-Vis photometer with a portable and Arduino microcontroller. Universidade Federal da Paraíba; 2014.

11. Koo H, Gomes BPFA, Rosalen PL, et al. In vitro antimicrobial activity of propolis and Arnica montana against oral pathogens. Archives of Oral Biology. 2000;45(2):141-148. 